The Journal of Laryngology \& Otology

http://journals.cambridge.org/JLO

Additional services for The Journal of Laryngology \& Otology:

Email alerts: $\underline{\text { Click here }}$

Subscriptions: $\underline{\text { Click here }}$

Commercial reprints: $\underline{\text { Click here }}$

Terms of use : $\underline{\text { Click here }}$

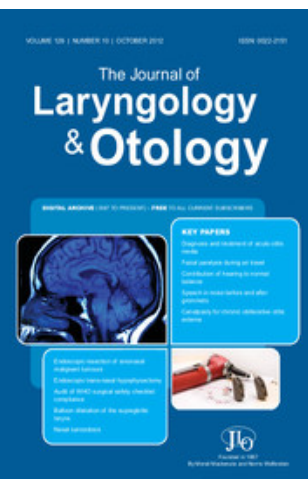

\title{
Adverse effects of nasal continuous positive airway pressure therapy in sleep apnoea syndrome
}

A. Kalan, G. S. Kenyon, T. A. R. Seemungal and J. A. Wedzicha

The Journal of Laryngology \& Otology / Volume 113 / Issue 10 / October 1999, pp 888 - 892

DOI: 10.1017/S0022215100145517, Published online: 29 June 2007

Link to this article: http://journals.cambridge.org/abstract_S0022215100145517

How to cite this article:

A. Kalan, G. S. Kenyon, T. A. R. Seemungal and J. A. Wedzicha (1999). Adverse effects of nasal continuous positive airway pressure therapy in sleep apnoea syndrome. The Journal of Laryngology \& Otology, 113, pp 888-892 doi:10.1017/ S0022215100145517

Request Permissions : $\underline{\text { Click here }}$ 


\title{
Adverse effects of nasal continuous positive airway pressure therapy in sleep apnoea syndrome
}

\author{
A. Kalan, F.R.C.S.(Ed.), G. S. KenYon, B.Sc., M.B.A., M.D., F.R.C.S., F.R.C.S.(ED.), \\ T. A. R. Seemungal, M.Sc., M.R.C.P., J. A. Wedzicha, M.A., M.D., F.R.C.P.
}

\begin{abstract}
Nasal continuous positive airway pressure (nCPAP) is now the treatment of choice for patients with sleep apnoea syndrome. Side-effects and adverse reactions have been described in isolated reports with this device. We have, therefore, systematically studied the side-effects of nCPAP therapy in 300 consecutive patients referred to the London Chest Hospital Ventilatory Support Unit. Ninety-six per cent of patients complained of at least one side-effect resulting from the therapy, with 45 per cent complaining of a sideeffect specific to the nasal mask. There was no correlation between the side-effects and level of pressure used during nCPAP. The rate of compliance remained high in spite of the side-effects, with a daily use of 7.8 hours (SD 8.05) and with 83 per cent of the patients using the device every night. Although nCPAP treatment remains acceptable to most patients there exists a high incidence of adverse effects.
\end{abstract}

Key words: Sleep apnoea syndromes

\section{Introduction}

Sleep apnoea syndrome (SAS) is defined by the occurrence of more than five apnoeas per hour of sleep (Guilleminault et al., 1978). Both cardiovascular and neuropsychological morbidity as well as considerable sleep disturbances have been demonstrated in patients with SAS who are untreated (Koskenvuo et al., 1987; Bedard et al., 1991) together with a higher risk of accidents while driving (Findley et al., 1988). There is, however, no effective pharmacological treatment for SAS (Strohl et al., 1986) and surgery such as uvulopalatopharyngoplasty (U3P) has been found of benefit in less than 50 per cent of unselected patients (Rodenstein, 1992). Only a few patients with severe SAS need referral for more advanced surgery (Riley et al., 1990). For these reasons, nasal continuous positive airways pressure is currently the first line therapy in moderate to severe SAS (Sullivan et al., 1981).

Nasal CPAP creates a positive pressure in the upper airways and provides a 'pressure splint' to eliminate apnoea (Kryger, 1994). It may also increase functional residual capacity and reflexly dilate the pharynx (Hoffstein et al., 1984). In SAS, nCPAP is usually considered when the apnoeahypopnoea index (the mean number of episodes of apnoea or hypopnoea per hour of sleep) is greater than 20. Although nasal CPAP is effective in reducing sleep apnoea syndrome it is frequently rejected by patients, partly because of the unwieldy and inconvenient nature of the treatment (Rauscher et al., 1991). Use of nCPAP is likely to be determined by the perceived benefits to the patient as well as the drawbacks of treatment, but the nature of these factors is not well understood.

Studies of CPAP use and its effects are highly variable in terms of patient selection and outcome measures employed (Engleman et al., 1994). CPAP compliance and acceptance is also variable with compliance dictated by side-effects in some studies and not in others (Kribbs et al., 1993). Even though CPAP is effective, compliance has been shown to vary from centre to centre and this may be due to staff skill and experience in initiating therapy, the adequacy of follow-up and perhaps, the type of CPAP device and mask used.

The present paper presents the results of a survey designed to study the adverse effects and the compliance with CPAP therapy in a large population. We believe our study has been performed on the largest patient sample to be conducted at a single centre.

\section{Methods}

All patients treated with nCPAP for a minimum of six months or more, at the London Chest Hospital over a 10-year period were included in the study. These patients were referred to the sleep laboratory 
with a suspicion of SAS and in all cases underwent a sleep study with chest wall movement, airflow and overnight oxygen saturation $\left(\mathrm{SaO}_{2}\right)$ monitoring prior to starting treatment. All had a diagnosis of OSA made by standard criteria followed by a nCPAP overnight trial. Almost all the patients were using second generation CPAP machines and silicone nasal masks as opposed to individually designed ones ('SOFTSTART', Devilbiss Ltd, West Midlands, UK. (85 per cent) and 'RESPIRONICS', Medic-Aid Ltd., West Sussex, UK. (15 per cent)). The pressure setting of the machine was adjusted to the level of pressure sufficient to abolish apnoeas and restore a correct sleep quality as assessed by repeat sleep studies.

All patients were regularly followed up in outpatients and machines serviced annually. Patients were advised to contact the clinical team if they experienced any problem with the machines. Faulty equipment was immediately replaced.

A questionnaire was sent to all the patients who had used nCPAP between January 1989 and November 1998 including those who had finished treatment at the time of the study. The questionnaire asked about compliance and periodicity of the use of nCPAP. Side-effects examined in detail included adverse effects of the nasal mask such as allergy, discomfort to the bridge of the nose, pain in the teeth or gums and air leaks near the eyes or over the face. We also enquired about dryness of the nose or mouth in the mornings on wakening, symptoms of sinusitis, sneezing, nasal drip, nasal congestion, nasal bleeding (epistaxis) and air swallowing (aerophagia). The questionnaire is detailed in Appendix I.

\section{Statistical analysis}

Categorical variables were binary coded for data analysis and inter-relationships explored by Spearman's correlations. Variables which showed significant univariate correlation with compliance were entered into a backward multiple logistic regression in order to determine which had independent effects on compliance.

\section{Results}

Of 354 patients issued with the questionnaires, 301 returned the completed forms ( 85.71 per cent). The majority of those who were non-responders had changed their address and could not be contacted. Included were 271 males and 30 females (mean age 54 years \pm 11.16 , range 26 years to 80 years). Two hundred and twenty-five patients (75 per cent) were obese with a mean BMI (body mass index) of 31.55 $\mathrm{kg} \mathrm{m}^{2}$ (range 24.09 to $41.17 \mathrm{~kg} \mathrm{~m}^{2}$ ).

The average self-reported daily use of $\mathrm{nCPAP}$ by the patients was $7.8 \pm$ four hours with 83 per cent of the patients using the device every night. This duration of use could not be independently corroborated as all the CPAP machines did not have an inbuilt electrical run-time recording facility. Eight patients (<three per cent) stated that they no longer used the machines and of these five gave no reason. Of the remaining three patients, two cited lack of benefit and the remaining one cited frequent awaken-

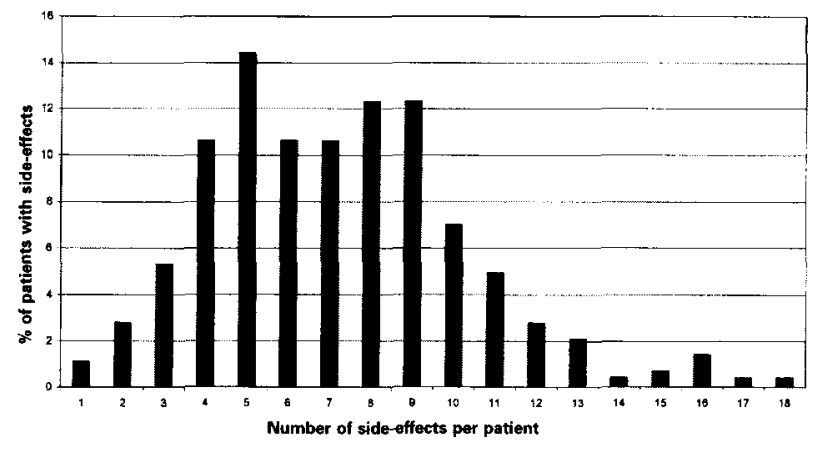

Fig. 1

Side-effects of nCPAP.

ings with use of the machine. Nine patients (three per cent) reported the use of nocturnal sedatives in order to tolerate the noise of the machine.

Two hundred and eighty-eight patients (96 per cent) complained of at least one side-effect resulting from the therapy. The percentage of patients suffering from adverse effects is summarized in Figure 1. The side-effects could be categorized as either being due to the nasal mask (Figure 2), to local intra-nasal adverse effects (Figure 3) or to problems due to the noise and bulk of the machines (Figure 4). In addition, 45 per cent of the patients complained of at least one side-effect due to the nasal mask (Figure 2) of which 162 (54 per cent) found the silicone mask uncomfortable and had it adjusted with benefit. Eighty-four (26.8 per cent) of the patients also reported allergic phenomena with the silicone mask and 156 (52 per cent) complained of pain or abrasion over the bridge of the nose. Seventy-eight (26 per cent) of the users also complained of air leaks from around the mask on to the eyes and the face leading to soreness of the eyes in the mornings.

One hundred and thirty-eight patients (46 per cent) also complained of dryness in the nose, while 210 (70 per cent) reported dryness of the mouth in the mornings. Eighty-seven (29 per cent) also complained of excessive sneezing, 102 (34.5 per cent) reported rhinorrhoea and 129 (43 per cent) experienced nasal blockage and congestion. Many users (108; 36 per cent) required some form of topically acting nasal steroid spray. Frank epistaxis was observed in only 0.9 per cent of the patients but blood-stained nasal mucus was reported in 51 (17 per cent). Aerophagia (leading to bloating and flatu-

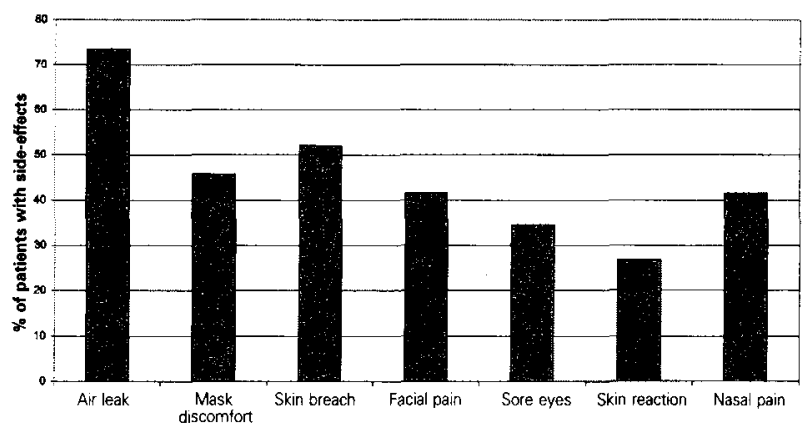

FIG. 2

Side-effects due to nasal mask. 


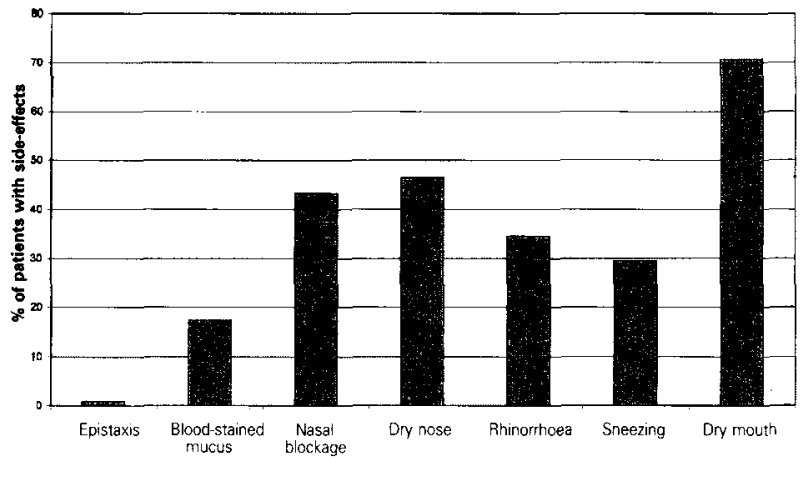

FIG. 3

Nasal side-effects.

lence) and symptoms suggestive of sinusitis was recorded in only a minority of cases $(0.4$ per cent).

The CPAP machine was considered too cumbersome and noisy by 135 (45 per cent) of the users causing many partners to sleep separately (39 per cent). One hundred and two (34 per cent) complained of sleep disturbances as a result of the loud noise.

The type of nCPAP machine used was not significantly related to the side-effects nor compliance. Compliance was reduced in patients reporting symptoms of dryness of the nose, leakage of air around the mask or disturbed sleep due to noisy equipment ( $p<0.02$ in all cases). Also, patients were less likely to use their nCPAP every night if they had any of the following side-effects (rho, p): bloodstained nasal mucus $(-0.18,0.002)$, noisy equipment $(-0.169,0.004)$, epistaxis $(-0.157,0.008)$ or if they slept separately $(-0.248,<0.001)$.

No life-threatening symptoms such as meningitis, pneumoencephaly or pneumothorax were seen, although these have been previously reported (Pepin et al., 1995).

\section{Discussion}

Sleep apnoea syndrome (SAS) is a life-threatening condition characterized by repetitive episodes of total or partial closure of the upper airways during sleep resulting in episodic hypoxaemia and arousal from sleep resulting in day-time somnolence. Nasal continuous positive airway pressure (nCPAP), introduced by Sullivan et al. in 1981, has become the treatment of choice for the initial management of SAS (Kryger, 1994). When used at night with an appropriate applied pressure, nasal CPAP eliminates

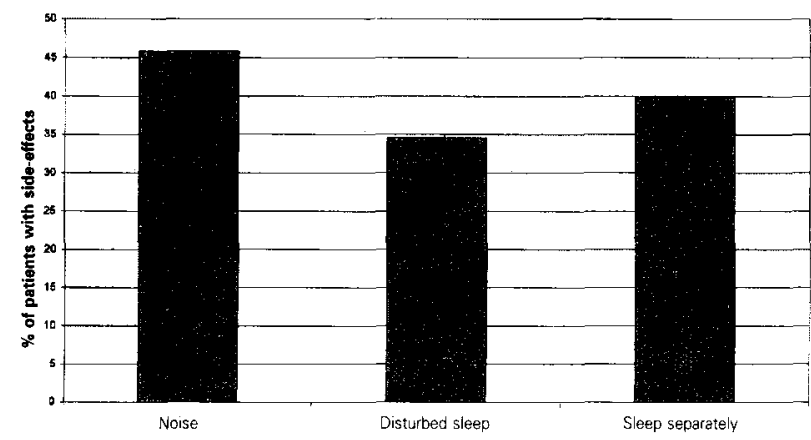

FIG. 4

Machine-related side-effects. episodes of apnoea and hypopnoea due to upper airways obstruction; the patient, therefore, enjoys more undisturbed sleep and less subjective hypersomnolence while awake (Rajagopal et al., 1986). CPAP has been shown to improve the long-term prognosis by preventing cardiopulmonary sequelae (Sullivan et al., 1981) although recent data does not support this relationship (Shiner et al., 1990; Hoffstein et al., 1991; Levinson and Millman, 1991).

In this study we have shown a high incidence of side-effects from the use of nCPAP. Adverse effects were principally related to intolerance of the nasal mask with the high rate of airflow leading to mucosal inflammation in the nose, dryness and aerophagia. The noise and bulk of the equipment was also a disincentive to continued use.

Local side-effects related to the nasal mask have been well described, both with nCPAP and intermittent positive pressure ventilation (NIPPV) using a nasal mask (Leger et al., 1992; Meecham Jones and Wedzicha, 1994). It would seem, however, that the type of mask used is of importance as reduced sideeffects have been reported in those using a tailormade mask. This is probably related to a better fit and to a reduction in air leaks. Therefore, education of the patient is important to obtain the best adjustment of the mask and to reduce air leakage.

The greatest side-effect seems to be related to high flow rates of air through the nose over long periods. In theory humidification could reduce these side-effects but in practice the use of humidification does not lead to reduced problems (Pepin et al., 1995). This was thought to be either due to inefficiency in the humidification systems presently available or to the lack of any effect from humidification whatever its technical efficiency. NIPPV seems to lead to a lesser degree of such side-effects, that may be due to the cessation of positive air flow in the expiratory phase of the respiratory cycle (Leger et al., 1992) or may be due to differing flow rates since higher flow rates are used with nCPAP compared with NIPPV (60 to 120 with nCPAP vs. 20 to 40 litres/minute with NIPPV).

Patients with SAS frequently complain of nasal obstruction. This has been hypothesized as a possible pre-existing condition leading to the development of SAS (Series et al., 1993). Such problems may, in turn, make the patients more susceptible to nasal sideeffects from the treatment compared with patients requiring CPAP who do not have as many preexisting nasal disorders.

We found that some of the patients had to use nocturnal sedatives for compliance despite contrary medical advice and these patients more frequently described problems with the noise of the machine. This use of sedatives was possibly linked with the poor quality of sleep, thus leading to a higher perception of side-effects but, in spite of this, only a few patients were unable to persist with the treatment. In our study, none of the patients required hospitalization because of serious sideeffects. 
Our study shows a similar level of side-effects to those reported by Hoffstein et al. (1992). However, recent data has suggested that the reported compliance may be much higher than the real compliance as others have found that only 46 per cent of patients met the criteria for regular use, defined as at least four hours of nCPAP administered on 70 per cent of the nights monitored (Kribbs et al., 1993). The same authors also found some association between non-physical side-effects (claustrophobia) and less regular use of nCPAP but they did not find any correlation between usage and the physical side-effects.

The more recent CPAP devices have improved design in that they are relatively quiet and are fitted with modern masks that are self-sealing, light and relatively comfortable. These improvements have greatly reduced complications such as noise intolerance, conjunctivitis due to air leaks and the ulceration of the nasal bridge due to mask pressure. In patients who cannot tolerate CPAP systems, adjustments that increase the pressure slowly as the patient falls asleep (the 'ramp device'), or those that reduce the expiratory pressure (BiPAP) may be helpful (Kryger, 1992). A damaged mask, increasing weight, alcohol use, nasal obstruction or very rarely machine failure can cause CPAP to fail late in the course of therapy (Sullivan and Grunstein, 1994).

In summary, when patients have no surgically remediable pathology, nCPAP is the method of choice for the treatment of moderate to severe SAS. Our study has shown that the incidence of side-effects from this therapy is high but not that limiting as the self-reported duration of use per night is excellent. The treatment nevertheless remains acceptable to most patients because of the derived benefits. Improved design of masks and machines will alleviate some of the difficulties in compliance but greater awareness of the nasal side-effects is required. More insight is also required as to the pathophysiology of these symptoms, so that effective treatment strategies can be designed to counteract them.

\section{Acknowledgements}

The authors would like to thank Christine Mikelsons, Superintendent Physiotherapist, London Chest Hospital for her guidance in drafting the questionnaire.

\section{References}

Bedard, M. A., Montplaisir, J., Richer, F. (1991) Nocturnal hypoxemia as a determinant of vigilance impairment in sleep apnoea syndrome. Chest 100: 367-370.

Engleman, H. M., Martine, S. E., Douglas, N. J. (1994) Compliance with CPAP therapy in patients with sleep apnoea/hypopnoea syndrome. Thorax 49: 263-266.

Findley, L. J., Unverzagt, M. E., Suratt, P. M. (1988) Automobile accidents involving patients with obstructive sleep apnoea. American Review of Respiratory Disease 138: 337-340.

Guilleminault, C., Van Den Hoed, J., Mitler, M. M. (1978) Clinical overview of the sleep apnoea syndrome. In Sleep Apnoea Syndromes. (Guilleminault, C., Dement, W. C., eds.), A. R. Liss, New York, pp 1-12.

Hoffstein, V., Chan, C. K., Slutsky, A. S. (1991) Sleep apnoea and systemic hypertension: a casual association. American Journal of Medicine 91: 190-196.
Hoffstein, V., Viner, S., Mateika, S. (1992) Treatment of obstructive sleep apnoea with nasal continuous positive pressure: Patient compliance, perception of benefits and side effects. American Review of Respiratory Disease 145: 841-845.

Hoffstein, V., Zamel, N., Phillipson, E. A. (1984) Lung volume dependence of pharyngeal cross-sectional area in patients with obstructive sleep apnoea. American Review of Respiratory Disease 130: 175-178.

Koskenvuo, M., Kaprio, J., Telakivi, T. (1987) Snoring as a risk factor for ischaemic heart disease and stroke in men. British Medical Journal 294: 16-19.

Kribbs, N. B., Pack, A. I., Kline, L. R. (1993) Objective measurement of patterns of nasal CPAP used by patients with obstructive sleep apnoea. American Review of Respiratory Disease 147: 887-895.

Kryger, M. H. (1994) Management of obstructive sleep apnoea: overview. In Principles and Practice of Sleep Medicine. 2nd Edition, (Kryger, M. H., Roth, T., Dement, W. C., eds.). W. B. Saunders, Philadelphia, pp 736-747.

Kryger, M. H. (1992) Management of obstructive sleep apnoea. Clinical Chest Medicine 13: 481-492.

Leger, P., Robert, D., Langwin, B. (1992) Chest wall deformities due to idiopathic kyphoscoliosis or sequelae of tuberculosis. European Respiratory Review 2: 362-368.

Levinson, P. D., Millman, R. P. (1991) Causes and consequences of blood pressure alterations in obstructive sleep apnoea. Archives of Internal Medicine 151: 455-462.

Meecham Jones, D. J., Wedzicha, J. A. (1994) Nasal masks for positive pressure ventilation: Survey of patient usage. Thorax 49: 811-812.

Pepin, J. L., Leger, P., Veale, D., Langevin, B., Robert, D., Levy, P. (1995) Side effects of nasal continuous positive airway pressure in sleep apnoea syndrome. Chest 107: 375-381

Rajagopal, K. R., Bennett, L. L., Dillard, T. A., Tellis, C. J., Tenholder, M. F. (1986) Overnight nasal CPAP improves hypersomnolence in sleep apnoea. Chest 90: 172-176.

Rauscher, H., Popp, W., Wanke, T. (1991) Acceptance of CPAP therapy for sleep apnea. Chest 100: 1019-1023.

Riley, R. W., Powell, N. B, Guilleminault, C. (1990) Maxillofacial surgery and nasal CPAP: a comparison of treatment for obstructive sleep apnoea syndrome. Chest 98: 1421-1425.

Rodenstein, D. O. (1992) Assessment of uvulopalatopharyngoplasty for the treatment of the sleep apnoea syndrome. Sleep 15: S52-S56.

Series, F., St Pierre, S., Carrier, G. (1993) Surgical correction of nasal obstruction in the treatment of mild sleep apnoea: Importance of cephalometry in predicting outcome. Thorax 48: $360-363$.

Shiner, R. J., Carroll, N., Sawicka, E. H., Simmond, A. K., Braithwaite, M. A. (1990) Role of nocturnal hypoxemia in the genesis of systemic hypertension. Cardiology 77: 25-29.

Strohl, K. P., Cherniack, N. S., Gothe, B. (1986) Physiologic basis of therapy for sleep apnoea. American Review of Respiratory Disease 134: 791-802.

Sullivan, C. E., Grunstein, R. R. (1994) Continuous positive airway pressure in sleep-disordered breathing. In Principles and Practice of Sleep Medicine, 2nd Edition, (Kryger, M. H., Roth, T., Dement, W. C., eds.). W. B. Saunders, Philadelphia, pp 694-705.

Sullivan, C. E., Issa, F. G., Berthon-Jones, M., Eves, L. (1981) Reversal of obstructive sleep apnoea by continuous positive airway pressure applied through the nose. Lancet 1: $862-865$.

Address for correspondence:

Mr A. Kalan, F.R.C.S. (Ed.)

11 Arrowhead Court,

James Lane,

Leytonstone

London E11 1NT.

Fax: 01815583134

e-mail: ak@kalan1.demon.co.uk 


\section{Appendix I-The Questionnaire}

Name

D.o.B.

Age

Sex

Please answer the following questions:

When did you start using your nCPAP machine?

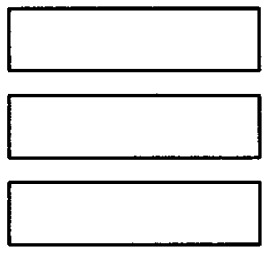

No. of hours used each night

Do you currently suffer from any of the following?

Nasal blockage

Dryness in nose

Nose bleeds

Pain over bridge of nose

Excessive sneezing

Blood stained mucus from nose

Runny nose

Dry mouth in the mornings

Dizziness

Sore eyes

Facial pains

Eczema

Hayfever

Asthma

Yes $\square$
Yes $\square$
Yes $\square$
Yes $\square$
Yes $\square$
Yes $\square$
Yes $\square$
Yes $\square$
Yes $\square$
Yes $\square$
Yes $\square$
Yes $\square$
Yes $\square$
Yes $\square$

No $\square$
No $\square$
No $\square$
No $\square$
No $\square$
No $\square$
No $\square$
No $\square$
No $\square$
No $\square$
No $\square$
No $\square$
No $\square$
No $\square$

Have you had any of the above problems in the past? (before starting CPAP treatment)

What is your experience of the following?

Noise of machine (Noisy)

Comfort of mask (Comfortable)

Air leaking from mask

Air leaking from your mouth

Skin reaction to mask

Breakdown of skin over nose (i.e. Pressure sore)

Have you taken any inhalers/spray for nasal stuffiness?

Do you sleep in a separate room to your spouse/partner? No

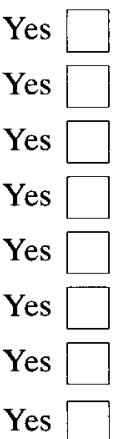

No No No No No

No No

No

Yes
No

Do you have sleep disturbance because of the nCPAP machine

Any other experiences/problems?

Thank you for your time and co-operation in this study. 